\title{
Vascular Territory Image Analysis Using Vessel Encoded Arterial Spin Labeling
}

\author{
Michael A. Chappell, Thomas W. Okell, Peter Jezzard, and Mark W. Woolrich \\ Oxford Centre for Functional MRI of the Brain, University of Oxford, \\ John Radcliffe Hospital, Headington, Oxford, OX4 3HD, UK \\ michael.chappell@clneuro.ox.ac.uk
}

\begin{abstract}
Arterial Spin Labeling (ASL) permits the non-invasive assessment of cerebral perfusion, by magnetically labeling all the blood flowing into the brain. Vessel encoded (VE) ASL extends this concept by introducing spatial modulations of the labeling procedure, resulting in different patterns of label applied to the blood from different vessels. Here a Bayesian inference solution to the analysis of VE-ASL is presented based on a description of the relative locations of labeled vessels and a probabilistic classification of brain tissue to vessel source. In simulation and on real data the method is shown to reliably determine vascular territories in the brain, including the case where the number of vessels exceeds the number of independent measurements.
\end{abstract}

\section{Introduction}

Arterial Spin Labeling (ASL) MRI is becoming an increasingly popular method for imaging cerebral blood flow (CBF), since it is both non-invasive and relatively rapid. ASL operates by labeling the water in blood magnetically via RF pulse inversion before entry to the brain and then imaging it as it passes into the capillaries. This 'tag' image is compared to a control image, taken in the absence of labeled blood, to remove the large static magnetization signal of the brain tissues.

More recently a number of methods have been proposed that use an ASL approach for Vascular Territory Imaging (VTI). The purpose of VTI is to identify the different regions of the brain that are supplied by individual feeding vessels. This type of imaging has a number of clinical applications, for example visualization of the vascular changes brought about by stroke or vascular stenosis. ASL based VTI can be achieved by making the ASL label spatially selective around individual vessels, for example [1, 2]. An alternative, but related, approach is Vessel Encoded (VE) ASL [3, 4]. In this method the strength of the inversion is modulated spatially across the labeling plane. A series of such images are collected each with different modulations chosen to uniquely encode each vessel's contribution.

VE-ASL is typically analyzed by assembling an encoding matrix that represents the effects of the different encoding steps on the vessels in the labeling plane. This matrix is then inverted to predict individual flow contributions in every brain voxel. In principle the encoding matrix is known a priori based on the experimental setup. However, due to head motion or imperfect alignment there may be some deviation in practice, leading to imperfect separation of vascular territories and inaccurate CBF estimates. 
Here we present a Bayesian solution to the analysis for VE-ASL data. Using the sinusoidal form of [5] we derive the relationship between vessel location and encoding matrix. A generative model based on this encoding matrix and a probabilistic classification of each voxel to a source vessel was constructed, using the assumption that voxels are typically fed by a single vessel. This method provides a unified approach to the analysis of VE-ASL combining the effects of vessel location on the encoding matrix with the estimation of vascular territories and local blood within a single adaptive algorithm.

\section{Methods}

The tag-control differencing of traditional ASL can be represented in any voxel in matrix form $\mathbf{y}=\mathbf{A x}$ :

$$
\mathbf{y}=\left[\begin{array}{l}
y_{1} \\
y_{2}
\end{array}\right] \quad A=\left[\begin{array}{cc}
-1 & 1 \\
1 & 1
\end{array}\right] \quad \mathbf{x}=\left[\begin{array}{l}
F \\
S
\end{array}\right],
$$

where $\mathbf{x}$ represents the sources of signal, $F$ being the signal from the flowing blood and $S$ being the static magnetization of the tissues; $\mathbf{y}$ is the vector of measured values, $y_{1}$ is the tag image $\left(y_{1}=S-F\right)$ and $y 2$ is the control $\left(y_{2}=S+F\right)$. This concept can be extended to VE-ASL with multiple sources of blood whose selection depends upon the spatial encoding. For example a three vessel encoding might look like (R, L and $\mathrm{B}$ - the right and left carotid, and basilar arteries respectively):

$$
\mathbf{y}=\left[\begin{array}{llll}
1 & 1 & 1 & 1 \\
-1 & -1 & -1 & 1 \\
1 & -1 & 0 & 1 \\
1 & 1 & -1 & 1
\end{array}\right]\left[\begin{array}{l}
R \\
L \\
B \\
S
\end{array}\right]
$$

Note the structure of the encoding matrix: the top row produces the control image, the second the tag image and there are two encoded tag images; the final column must always be all unity values, since this dictates the contribution of the static magnetization of the tissues to the measured signal that appears in all images.

It is possible to find the contributions from each vessel in every brain voxel by matrix inversion: $\mathbf{x}=\mathbf{A}^{-1} \mathbf{y}$. In practice the encoding matrix will not be square, thus the matrix pseudo-inverse will be required. Hence this method provides a solution optimal according to a least-squares cost function.

To account for any encoding setup and allow for misalignment between vessel locations and spatial modulation of the tag, we derived a general encoding matrix based on the sinusoidal modulation assumption [4]. Each vessel encoded ASL image is the result of labeled blood arriving in the tissue from multiple source vessels. The extent of the inversion of the blood in each vessel is set by the spatial modulation of the applied tagging and is thus determined by the relative geometry between the vessel and the spatial modulation. The spatial modulation occurs across the tagging plane, although the variation in inversion is only prescribed in one direction at a time, for example modulation parallel to the $\mathrm{x}$-axis. Each spatial modulation may thus be defined in terms of a modulation centre, $\left(c_{x}, c_{y}\right)$, and a modulation angle, $\theta$, relative to a 
Cartesian axis placed in the tagging plane. Each vessel/source can be defined in terms of its location in the tagging plane, $(x, y)$, relative to the same axes, as illustrated in Fig. 1. The inversion state of each vessel during the acquisition of a single encoded image is determined by its distance, $d$, from the modulation centre and the form of the spatial modulation, $f: m=f(d)$, where from Fig. 1:

$$
d= \begin{cases}\sqrt{\left(x-c_{x}\right)^{2}+\left(y-c_{y}\right)^{2}} \cos \left(\theta-\arctan \left(\frac{y-c_{y}}{x-c_{x}}\right)\right) & x-c_{x}>0 \\ \sqrt{\left(x-c_{x}\right)^{2}+\left(y-c_{y}\right)^{2}} \cos \left(\theta-\arctan \left(\frac{y-c_{y}}{x-c_{x}}\right)+\pi\right) & x-c_{x}<0 \\ \left(y-c_{y}\right) \cos (\theta-\pi / 2) & x-c_{x}=0, y-c_{y}>0 \\ \left(y-c_{y}\right) \cos (\theta+\pi / 2) & x-c_{x}=0, y-c_{y}<0\end{cases}
$$

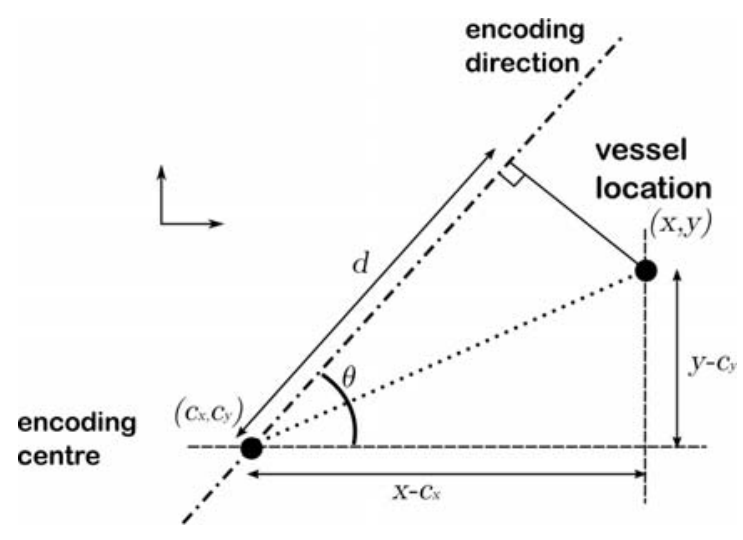

Fig. 1. The geometry for the spatial encoding of a vessel

The spatial encoding function may be approximated by a sinusoidal form [5] $m=\sin (\pi d / 2 D)$, where $D$ is the spatial scale along the encoding direction. This form represents the periodic nature of the actual encoding applied. In a vessel encoding experiment a number of encoded images will be acquired. Hence there will be a modulation value for each combination of vessel and modulation phase, i.e. for the $i$ th encoded image and $j$ th vessel $m_{i j}=f\left(\mathbf{x}_{j}, \mathbf{c}_{i}, \theta_{i}, D_{i}\right)$. This modulation value corresponds to an entry in a general encoding matrix:

$$
\mathbf{E}=\left[\begin{array}{lllll}
1 & 1 & \cdots & 1 & 1 \\
-1 & -1 & \cdots & -1 & 1 \\
m_{11} & m_{12} & \cdots & m_{1 M} & 1 \\
\vdots & \vdots & \ddots & \vdots & 1 \\
m_{N 1} & m_{N 2} & \cdots & m_{N M} & 1
\end{array}\right]
$$

This form of encoding matrix permits each voxel to be fed by every vessel. In practice vascular territories are relatively well defined, such that most vessels will only be 
sourced from a single vessel. This was incorporated into the forward model by way of a classification matrix, such that in voxel $k$ the vector of signal intensities arising from the blood flow from vessel $c$ was given by $\mathbf{s}_{k, c}=\mathbf{E P}_{c} \mathbf{f}_{k}$, where $\mathbf{f}_{k}=\left[\begin{array}{ll}f_{k} & s_{k}\end{array}\right]^{\mathrm{T}}$ with flow, $f_{k}$, and static signal $s_{k}, \mathbf{E}$ is the encoding matrix based on vessel locations $\mathbf{x}_{j} \in\{\mathbf{X}: j=1,2, \ldots, M\}$ (and known encoding setup) and $\mathbf{P}_{\mathrm{c}}$ is the classification matrix that selects vessel $c$, which is a $(M+1) \times 2$ matrix with all elements set to zero except $P_{c, 1}=1$ and $P_{M+1,2}=1$.

Assuming Gaussian noise the Likelihood was formed:

$$
\begin{aligned}
& \operatorname{Pr}(\mathbf{Y} \mid \mathbf{F}, \mathbf{X}, \mathbf{q}=\kappa, \pi, \phi)=\prod_{k} \operatorname{Pr}\left(\mathbf{y}_{k} \mid q_{k}=\kappa_{k}, \mathbf{f}_{k}, \mathbf{X}, \phi_{k}\right), \\
& \operatorname{Pr}\left(\mathbf{y}_{k} \mid \mathbf{f}_{k}, \mathbf{X}, q_{k}=\kappa_{k}, \pi_{\kappa_{k}}, \phi_{k}\right)=\left(\phi_{k}^{N / 2} /(2 \pi)^{N / 2}\right) e^{-\frac{\phi_{k}}{2}\left(\mathbf{y}_{k}-\mathbf{s}_{k, \kappa_{k}}\right)^{T}\left(\mathbf{y}_{k}-\mathbf{s}_{k, \kappa_{k}}\right)},
\end{aligned}
$$

where $\mathbf{q}$ is the map of discrete class labels and $\kappa$ is a specific configuration thereof, $\phi_{k}$ is the noise precision and $\pi_{c}$ is the proportion of voxels belonging to vessel $c$. Application of Bayes' theorem gave the posterior distribution:

$$
\operatorname{Pr}(\mathbf{F}, \mathbf{X}, \mathbf{q}=\kappa, \pi, \phi \mid \mathbf{Y}) \propto \operatorname{Pr}(\mathbf{Y} \mid \mathbf{F}, \mathbf{X}, \mathbf{q}=\kappa, \phi) \operatorname{Pr}(\mathbf{F}, \mathbf{X}, \phi) \operatorname{Pr}(\mathbf{q}=\kappa \mid \pi) \operatorname{Pr}(\pi),
$$

where the following priors were chosen:

$$
\begin{gathered}
\operatorname{Pr}(\mathbf{F}, \mathbf{X}, \phi)=\operatorname{Pr}(\mathbf{F}) \operatorname{Pr}(\mathbf{X}) \operatorname{Pr}(\phi), \\
\operatorname{Pr}(\mathbf{F})=\text { Uniform, } \operatorname{Pr}(\phi)=\phi^{-1}, \operatorname{Pr}(\mathbf{X})=N\left(\mathbf{X}_{0}, 0.1\right),
\end{gathered}
$$

where vessel locations were normalized to scale between -1 and 1 and $\mathbf{X}_{0}$ are the vessel locations estimated from the ideal encoding:

$$
\operatorname{Pr}\left(q_{k}=c \mid \pi\right)=\pi_{c} \text {, with } \sum \pi_{c}=1, \operatorname{Pr}\left(\pi_{c}\right)=\operatorname{Uniform}(0,1) .
$$

For the purposes of inference we marginalized analytically over both the flow and class label in every voxel:

$$
\operatorname{Pr}(\mathbf{X}, \pi, \phi \mid \mathbf{Y}) \propto \prod_{k}\left[\phi_{k}^{-1} \sum_{c}\left\{\pi_{c}\left|\mathbf{P}_{c}^{T} \mathbf{E}^{T} \mathbf{E} \mathbf{P}_{c}\right| e^{-\frac{\phi}{2}\left(\mathbf{y}_{k}-\mathbf{E} \mathbf{E}_{c}\left(\mathbf{P}_{c}^{T} \mathbf{E}^{T} \mathbf{E P}_{c}\right)^{-1} \mathbf{P}_{c}^{T} \mathbf{E}^{T} \mathbf{y}_{k}\right)}\right\}\right] \operatorname{Pr}(\mathbf{X}) \operatorname{Pr}(\pi) .
$$

The resulting marginal distribution was inferred using Metropolis Hastings (MH) and point estimates for $\mathbf{X}, \pi$ and $\phi$ were determined. These point estimates were then used to determine the flow in every voxel from each vessel.

\section{Results}

Analysis was performed on simulated data to establish the ability of the method to infer vessel locations and flow contributions from VE-ASL data. Four source vessels were defined with flow contributions as in Fig. 2 (top). To simplify the interpretation (and for comparison with real data later) the vessels were all assumed to lie on the $\mathrm{x}$ axis with locations: $-1.1,0.9,-0.2,0.1$. The encoding setup reflected this restriction employing modulation only in the x-direction, as shown in Table 1. 


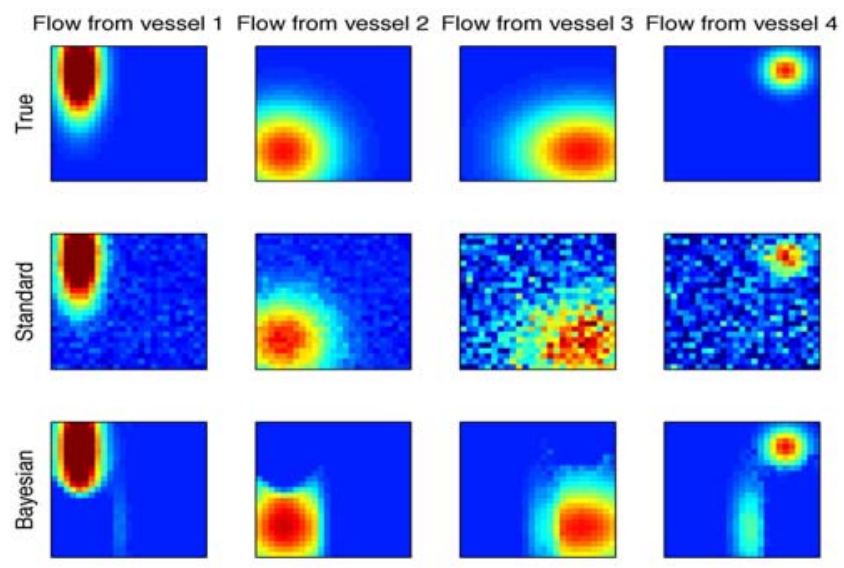

Fig. 2. Four-vessel simulation showing contributions to the flow from each vessel as separate columns. Top row: true simulated flow maps; middle row: results of standard matrix inversion analysis, using true vessel locations; bottom row: results of Bayesian classification model.

Table 1. Four vessel simulated data encoding setup

\begin{tabular}{llll}
\hline Cycle & c & $\theta^{\circ}$ & $D$ \\
\hline 1 & $(0,0)$ & 0 & 1 \\
2 & $(-0.5,0)$ & 0 & 0.5 \\
3 & $(0.5,0)$ & 0 & 1 \\
\hline
\end{tabular}

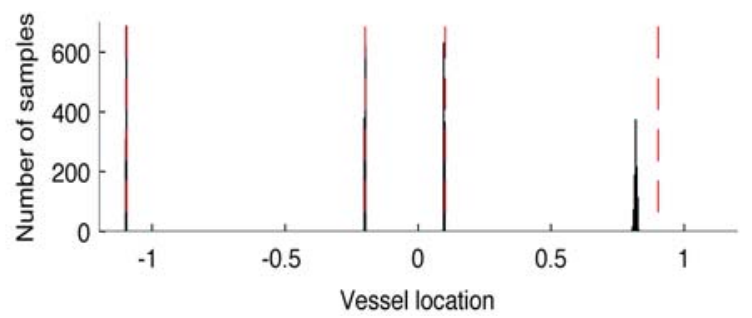

Fig. 3. MH samples for vessel locations, true locations superimposed (red dashed lines)

A standard analysis was performed using the correct vessel locations to form the encoding matrix, the individual flow maps are shown in Fig. 2 (middle row). The Bayesian analysis was initialized with 'ideal' vessel locations of $-1,1,-0.1,0.1$ and the resulting flow maps are shown in Fig. 2 (bottom row). The standard analysis was able to separate out the 4 vessel's contributions. However, the flow estimates were visibly noisy, a result of the encoding matrix being rank deficient, since the number of independent encodings is less than the number of sources. Unlike the standard analysis the Bayesian approach was also able to estimate the vessel locations, as shown in Fig. 3. It was able to give more accurate estimates of $\mathrm{CBF}$ and, for the majority of the voxels, correctly identify the main source vessel. There was some ambiguity in the contribution from vessels 3 and 4 . The probabilistic classification was not able to fully model the substantial overlap between the territories of the simulated vessels because it sought to classify each voxel to a specific vessel source. 
Table 2. Encoding setup for the first real dataset

\begin{tabular}{llll}
\hline Cycle & $\mathbf{C}$ & $\theta^{\circ}$ & $D$ \\
\hline 1a & $(0,0)$ & 0 & 1 \\
1b & $(0,0)$ & 180 & 1 \\
2a & $(-0.5,0)$ & 0 & 0.5 \\
2b & $(-0.5,0)$ & 180 & 0.5 \\
\hline
\end{tabular}

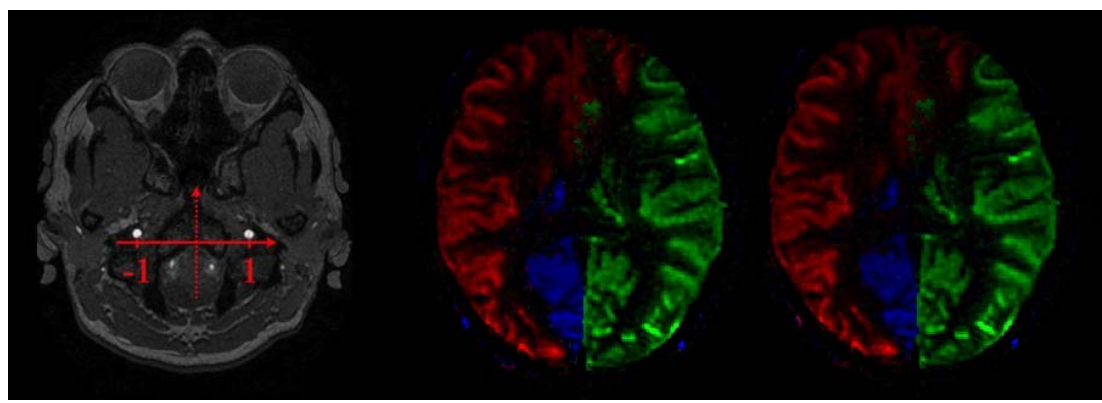

Fig. 4. Vessel encoding of the carotid and vertebral vessels. Angiographic image of the tagging plane (left) with encoding geometry imposed. Vascular territory map from the threevessel analysis using standard matrix inversion (middle) and Bayesian method (right), intensity represents $\mathrm{CBF}$, colour coding indicates dominant source vessel.

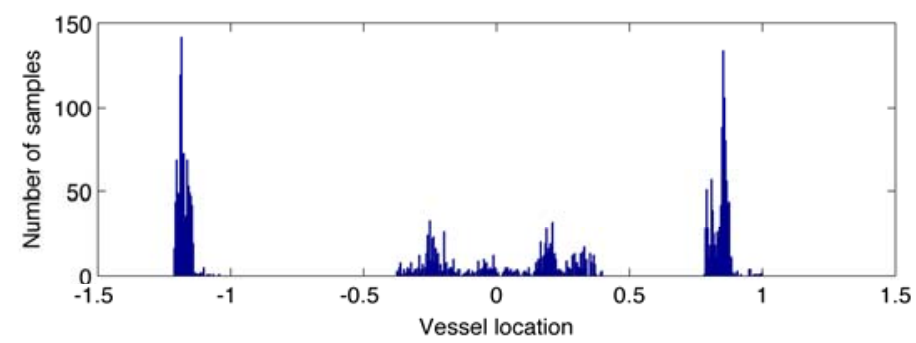

Fig. 5. MH samples for the estimation of vessel location for the first set of real data

The Bayesian analysis method was applied to a single slice of real vessel encoded data. This data was encoded following a similar one-dimensional setup to the simulated data used above, as shown in Table 2. Whilst four cycles were used to collect the data, there are only two truly independent cycles in this case.

The tagging plane for this subject is shown in Fig. 4 (left) and the encoding was arranged such that the vessels should fall at locations $-1,0,1$, the two vertebral arteries being regarded as a single vessel. The results of a standard analysis taking these vessel locations is shown in Fig. 4 (middle). The Bayesian classification analysis estimated a VTI map as in Fig. 4 (right), there was very good agreement between the estimated VTI images from both methods. The estimated locations for the Bayesian analysis are given in Fig. 5, they tend to imply a slight mis-alignment between the 


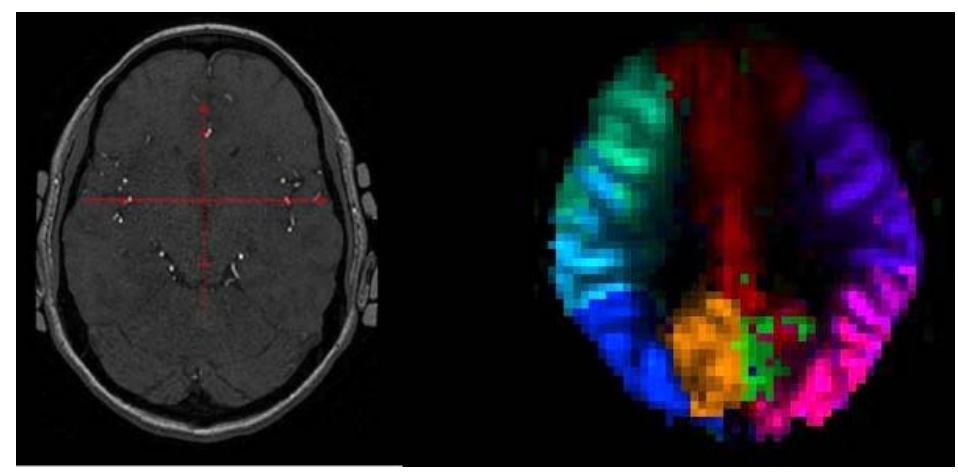

Fig. 6. Vessel encoding above the 'Circle of Willis'. Angiographic image of the tagging plane (left) with encoding geometry. Vascular territory map from the Bayesian analysis (right), intensity represents $\mathrm{CBF}$, colour coding indicates dominant source vessel.

left-right encoding and the carotid arteries. The results also suggest that both the vertebral arteries feed the posterior territory, since there is a mode either side of zero in locations consistent with the vertebral locations in Fig. 4. This is consistent with the physiology since the two vertebral arteries merge to become the basilar above the tagging plane chosen here.

Finally the Bayesian method was applied to a more complex scenario when tagging above the Circle of Willis. The tagging plane is shown in Fig. 6 (left) and 12 encodings (6 independent) were collected, 4 left-right and 8 anterior-posterior. From the angiographic image 11 'vessels' were identified, where for practicality some very closely space vessels were merged. The Bayesian method was able to identify unique territories for 9 of the vessels, Fig. 6 (right), this is simply not possible using encoding matrix inversion. The two pairs of posterior vessels were not separable, implying that they were not uniquely tagged due to the thickness of the tagging region.

\section{Discussion}

A Bayesian approach to the analysis of VE-ASL data has been presented. Having defined the geometrical relationship between vessels in the tagging plane and the encoding geometry it is now possible to specify the correct encoding matrix for any dataset. Alongside this, within the Bayesian inference it is possible to account for subject movement or misalignment by determining the exact vessel locations from the data. The inference of vessel locations will also be correcting for disagreement between the assumed sinusoidal modulation of the tag and the true function achieved in practice. The true form can be determined theoretically [4] and could be incorporated into the model. It has been shown the modulation function itself will vary with the flow velocity in the tagged vessel and it would be possible to incorporate this effect within the model introducing a further 'flow speed' parameter.

The presented analysis approach uses a probabilistic classification between voxels and the source vessels permitting analysis of data that contains greater vessels than there are independent encodings. This classification approach makes the assumption 
that each voxel is fed by only single vessel. The 'soft' probabilistic form of the classification makes some allowance for mixing, as might be expected in the 'watershed regions' at the borders of the vascular territories. However, simulations suggest it is not very accurate at estimation in regions of extensive mixing. For example the posterior territory is fed by the basilar artery, which is the result of merging of the two vertebral arteries and was seen in the first real dataset (Fig. 4). The classification approach would be unable to accurately model this, though neither would the standard matrix inversion approach be appropriate in that situation. It would be feasible to extend the classification to permit two (or more) sources per voxel to more accurately assess mixing in 'watershed' regions. It is also possible to define a full 'partial-flow' model by removing the classification matrix, whilst it is only possible to infer with this model if the number of encoding cycles at least equals the number of vessels. If such data were available this would provide a true ability to analyze mixed vascular territories.

Acknowledgments. The authors thank Eric Wong for provision of VE-ASL data.

\section{References}

1. Davies, N.P., Jezzard, P.: Selective Arterial Spin Labeling (SASL): Perfusion Territory Mapping of Selected Feeding Arteries Tagged Using Two-Dimensional Radiofrequency Pulses. Magn. Reson. Med. 49, 1133-1142 (2003)

2. Hendrikse, J., van der Grond, J., Lu, H., van Zijl, P.C.M., Golay, X.: Flow Territory Mapping of the Cerebral Arteries with Regional Perfusion MRI. Stroke 35, 882-887 (2004)

3. Gunther, M.: Efficient Visualisation of Vascular Territories in the Human Brain by Cycled Arterial Spin Labeling MRI. Magn. Reson. Med. 56, 671-675 (2006)

4. Wong, E.C.: Vessel-Encoded Arterial Spin-Labeling Using Pseudocontinuous Tagging. Magn. Reson. Med. 58, 1086-1091 (2007)

5. Wong, E.C., Kansagra, A.: Mapping Middle Cerebral Artery Branch Territories with Vessel Encoded Pesudo-Continuous ASL: Sine/Cosine Tag Modulation and Data Clustering in Tagging Efficiency Space. In: Proc. ISMRM, Toronto (2008) 